\title{
On Existence and Asymptotic Behaviour of Solutions of a Nonlinear Stochastic Integral Equation (*).
}

\author{
DOMINIK SzYNAI - STANISEAW WFERTYCHOWICZ
}

Summary. - The aim of the paper is to study a nonlinear stochastic integral equation of the form

$$
x(t ; \omega)=h(t, x(t ; \omega))+\int_{0}^{t} k_{1}(t, \tau ; \omega) f_{1}(\tau, x(\tau ; \omega)) d \tau+\int_{0}^{t} k_{2}(t, \tau ; \omega) f_{2}(\tau, x(\tau ; \omega)) d \beta(\tau ; \omega) .
$$

Sufficient conditions are given for the existence of random solutions and their asymptotic behaviour. The method of the fixed-point theorem of Darbo type-used in this paper, allowed us to generalize the results of [7].

\section{1. - Introduction.}

Random differential and integral equations play an important role in characterizing of many social, physical, biological and engineering problems. Theoretical and applicable treatments of problems concerning random differential and integral equations can be found in many papers and monographs (cf. [3], [4], [6], [7], [8], $[9],[10],[11],[12],[13],[14])$. The goal of this paper is to give a new existence theorem for a nonlinear stochastic integral equation treated in [7] and to investigate its asymptotic behaviour. Moreover, we present an application of our results to control systems. The considerations of this paper are based on the notion of a measure of noncompactness in Banach space and the fixed-point theorem of DARBo type [5], [2]. In our procedare, we construct first the real Banach space of tempered. functions and next define measures of noncompactness on that space where we are seeking solutions of the considered equation. This approach allows us to find weaker conditions than those of [7]; for instance, we replace the Lipschitz type conditions by those with sublinear functions. Moreover, we are able to investigate the behaviour of solutions at infinity.

We shall deal with a stochastic functional-integral equation of the form

$$
\begin{aligned}
x(t ; \omega)=h(t, x(t ; \omega))+\int_{0}^{t} k_{1}(t, \tau ; \omega) f_{1}(\tau, x(\tau ; \omega)) d \tau+ & \\
& +\int_{0}^{t} k_{2}(t, \tau ; \omega) f_{2}(\tau, x(\tau ; \omega)) d \beta(\tau ; \omega)
\end{aligned}
$$

(*) Entrata in Redazione il 19 giugno 1984; versione riveduta il 20 ottobre 1984.

Indirizzo degli A.A.: Instytut Matematyki UMCS, ul. Nowotki 10, 20-031 Lublin, Poland. 
where

(i) $\omega \in \Omega$, the supporting set of a complete probability measure space, $(\Omega$, $\mathcal{A}, P$ ), with $\mathcal{A}$ being the $\sigma$-algebra and $P$ the probability measure;

(ii) $x(t ; \omega)$ is the unknown random process;

(iii) $h(t, x)$ is a map from $R_{+} \times R$ into $R$;

(iv) $k_{1}(t, \tau ; \omega)$ and $k_{2}(t, \tau ; \omega)$ are the stochastic kernels which are random valued functions defined for $0 \leqslant \tau \leqslant t<\infty$ and $\omega \in \Omega$;

(v) $f_{1}(\dot{t}, x)$ and $f_{2}(t, x)$ are maps from $R_{+} \times R$ into $R$;

(vi) $t \in R_{+} \equiv[0, \infty)$ and $\beta(t ; \omega)$ is a stochastic process.

The first part of the stochastic system (1.1) is to be understood as an ordinary Lebesgue integral with probabilistic characterization, while the second part is an Ito-Doob stochastic integral.

\section{2. - Mathematical preliminaries.}

We shall give here some of the important mathematical concepts that are essential in understanding the details of this paper.

With respect to the random process $\beta(t ; \omega)$ we shall assume that for each $t \in R_{+}$, a minimal $\sigma$-algebra $\mathfrak{A}_{t}, \mathfrak{A}_{t} \subset \mathcal{A}$, is defined such that $\beta(t ; \omega)$ is measurable with respect to $\mathfrak{A}_{i}$. In addition, we shall assume that the minimal $\sigma$-algebra $\mathcal{A}_{t}$ is an increasing family such that

(i) the random process $\left\{\beta(t ; \omega), \mathcal{A}_{t}: t \in R_{+}\right\}$is a real martingale and

(ii) there is a real continuous non-decreasing function, $F(t)$, such that for $s<t$ we have

$$
E\left\{|\beta(t ; \omega)-\beta(s ; \omega)|^{2}\right\}=E\left\{|\beta(t ; \omega)-\beta(s ; \omega)|^{2} \mid \mathcal{A}_{t}\right\}=F(t)-F(s), \quad P \text {-a.ө. }
$$

where $E$ denotes the expected value of the random process.

We shall denote by $L^{2}(\Omega, \mathcal{A}, P)$ the space of $\mathcal{A}$ measurable square integrable $\operatorname{maps} x(t ; \cdot)$ with

$$
|x(t)|_{L^{2}}=E^{\frac{1}{2}}|x(t)|^{2}=\left(\int_{\Omega}|x(t ; \omega)|^{2} d P(\omega)\right)^{\frac{1}{2}}
$$

DEFINITION 2.1. - We shall call $x(t ; \omega)$ a random solution of the stochastic integral equation (1.1) if for every fixed $t \in R_{+}, x(t ; \omega) \in L^{2}(\Omega, A, P)$ and satisfies (1.1) $P$-a.e. 
Throughout this paper $\mathfrak{X}$ will denote an infinite dimentional real Banach space with norm \|\| and the zero element $\theta . K(x, r)$ stands for the closed ball centered at $x$ with radius $r$. Denote by $\mathcal{M}_{x}$ the family of all nonempty bounded subsets of $X$, and by $\mathcal{N}_{\mathfrak{X}}$ the family of all. relatively compact and nonempty subsets of $\mathfrak{X}$.

The axioms defining a measure of noncompactness are taken from [2].

DefintTion 2.2. - A nonempty family $\mathfrak{S} \subset \mathcal{N}_{\mathfrak{X}}$ is said to be the kernel (of a measure of noncompactness), provided it satisfies the following conditions:

$$
\begin{aligned}
& 1^{\circ} \quad U \in \mathfrak{T} \Rightarrow \bar{U} \in \mathfrak{T} ; \\
& 2^{\circ} \quad U \in \mathfrak{T}, V \subset U, V \neq \emptyset \Rightarrow V \in \mathfrak{T} ; \\
& 3^{\circ} \quad U, F \in \mathfrak{T} \Rightarrow \lambda U+(1-\lambda) V \in \mathfrak{T}, \lambda \in[0,1] \\
& 4^{\circ} \quad U \in \mathfrak{T} \Rightarrow \text { Conv } U \in \mathfrak{T} ; \\
& 5^{\circ} \quad \mathfrak{T}^{c} \text { (the subfamily of } \mathfrak{T} \text { consisting of all closed sets) is closed in } \mathscr{M}_{\mathfrak{C}}^{e} \text { with }
\end{aligned}
$$
respect to the Hausdorff topology.

Definttion 2.3. - The function $\mu: \mathscr{A}_{X} \rightarrow[0,+\infty)$ is said to be a measure of noncompactness with the kernel $\mathfrak{T}(\operatorname{ker} \mu=\mathfrak{T})$ if it is subject to the following conditions:

$$
\begin{aligned}
& 1^{\circ} \mu(U)=0 \Leftrightarrow U \in T ; \\
& 2^{\circ} \mu(U)=\mu(\bar{U}) ; \\
& 3^{\circ} \mu(\operatorname{Conv} U)=\mu(U) ; \\
& 4^{0} \quad U \subset V \Rightarrow \mu(U) \leqslant \mu(V) ; \\
& 5^{0} \mu(\lambda U+(1-\lambda) V) \leqslant \lambda \mu(U)+(1-\lambda) \mu(V), \quad \lambda \in[0,1] ; \\
& 6^{\circ} \text { if } U_{n} \in \mathscr{M}_{x}, U_{n}=\bar{U}_{n} \text { and } U_{n+1} \subset U_{n}, n=1,2, \ldots, \text { and if } \lim _{n \rightarrow \infty} \mu\left(U_{n}\right)=0, \\
& \quad \text { then } U=\bigcap_{n=1}^{\infty} U_{n} \neq \emptyset .
\end{aligned}
$$

If a measure of noncompactness $\mu$ satisfies in addition the following two conditions:

$$
\begin{aligned}
& 7^{\circ} \mu(U+V) \leqslant \mu(U)+\mu(V) ; \\
& 8^{\circ} \mu(\lambda U)=|\lambda| \mu(U), \quad \lambda \in R ;
\end{aligned}
$$

it will be called sublinear.

Let $M \subset \mathfrak{X}$ be a nonempty set and $\mu$ a measure of noncompactness on $X$.

Definimion 2.4. - We say that a mapping $T: M \rightarrow X$ is a contraction with respect to $\mu$ ( $\mu$-contraction) if for any set $U \in \mathcal{M}_{x}$ its image $T U \in \mathcal{M}_{X}$, and there exists a constant $k \in[0,1)$ such that

$$
\mu(T U) \leqslant k \mu(U)
$$


We shall use the following modified version of the fixed-point theorem of Darbotype.

THEOREir 2.1 [2]. - Let $O$ be a nonempty, closed, convex and bounded set of $X$, and let $T: O \rightarrow O$ be an arbitrary $\mu$-contraction. Then $T$ has at least one fixed point and the set Fix $T=\{x \in C: T x=x\}$ of fixed points of $T$ belongs to ker $\mu$.

Let $p(\cdot)$ be a positive continuous function defined on $[0,+\infty)$ such that $\lim _{T \rightarrow \infty} \sup _{t \geqslant T} p(t)=0$.

By $C_{p}\left(R_{+}, L^{2}(\Omega, A, P), p\right)$ (or shortly $\left.C_{p}\right)$ we denote a space of all continuous maps $x(t ; \cdot)$ from $R_{+}$into $L^{z}(\Omega, \mathcal{A}, P)$ with the topology defined by the norm

$$
\|x\|_{p}=\sup \left[p(t)\left\|_{x}(t)\right\|_{L^{2}}: t \geqslant 0\right]<\infty
$$

The space $O_{p}$ with norm \|\|$_{p}$ is a real Banach space (cf. $[1],[1 \tilde{5}]$ ).

Now for fixed $x \in O_{p}, U \in \mathbb{M}_{C_{p}}, T>0$, and $\varepsilon>0$, we put

$$
\begin{aligned}
& \beta^{T}(\mathscr{x}, \varepsilon)=\sup \left[\|p(t) x(t)-p(s) x(s)\|_{L^{2}}: t, s \in[0, T],|t-s| \leqslant \varepsilon\right], \\
& \beta^{T}(U, \varepsilon)=\sup \left[\beta^{T}(x, \varepsilon): x \in U\right] \\
& \beta_{0}^{T}(U)=\lim _{\varepsilon \rightarrow 0} \beta^{T}(U, \varepsilon) \\
& \beta_{0}(U)=\lim _{T \rightarrow \infty} \beta_{0}^{T}(U) \\
& a(U)=\lim _{T \rightarrow \infty} \sup _{x \in U}\left[\sup _{t \geqslant T} p(t)\|x(t)\|_{L^{2}}\right] \\
& \bar{b}(U)=\lim _{T \rightarrow \infty} \sup _{x \in D}\left[\sup \|p(t) x(t)-p(s) x(s)\|_{L^{2}}\right] \\
& \mu_{0}(U)=\beta_{0}(U)+a(U)+\sup [p(t) m(U(t)): t \geqslant 0] \\
& b_{1}(U)=\beta_{0}(U)+b(U)+\sup [p(t) m(U(t)): t \geqslant 0]
\end{aligned}
$$

where $m$ is a sublinear measure of noncompactness on $\mathscr{K}_{L^{2}(\Omega, \mathcal{A}, P)}$, and $U(t)=[x(t) \in$ $\left.\in L^{2}(\Omega, \mathfrak{A}, P): \infty \in U\right]$.

The functions $\mu_{0}$ and $\mu_{1}$ define sublinear measures of noncompactness on $\mathscr{M}_{C_{p}}$ ([1], [2]). It is also known ([1], [2]) that ker $\mu_{0}$ is the set of all sets $U \in \mathcal{N}_{C_{p}}$ such that the functions belonging to $U$ are equicontinuous on any compact of $R_{+}$and

$$
\lim _{t \rightarrow \infty} p(t)\|x(t)\|_{L^{2}}=0
$$

uniformly with respect to $x \in U$. Further properties of $\mu_{0}$ and $\mu_{1}$ can be found in [1] and [2]. 


\section{3. - Main results.}

Let $t \in R_{+}$be fixed. We suppose that the stochastic kernels $k_{1}(t, \tau ; \omega)$ and $k_{2}(t, \tau ; \omega)$ are measurable for each, $0 \leqslant \tau \leqslant t, P$-essentially bounded and continuous as maps from the set

$$
\Delta \equiv\{(t, \tau): 0 \leqslant \tau \leqslant t<\infty\} \quad \text { into } L_{\infty}(\Omega, \mathcal{A}, P) .
$$

Define for $0 \leqslant \tau \leqslant t<\infty$,

$$
\left\|k_{i}(t, \tau)\right\|=P-\operatorname{ess} \sup _{\omega \in \Omega}\left|k_{i}(t, \tau ; \omega)\right|, \quad i=1,2 .
$$

The above assumptions imply that if $x \in C_{p}$, then for each $t \in R_{+}$

$$
\left\|k_{t}(t, \tau) x(\tau)\right\|_{L^{2}} \leqslant\left\|k_{i}(t, \tau)\right\|\|x(\tau)\|_{L^{2}}, \quad i=1,2
$$

In what follows we shall often use the following estimate

$$
\left\|\int_{0}^{t} k_{2}(t, \tau) f_{2}(\tau, x(\tau)) d \beta(\tau)\right\|_{L^{2}} \leqslant\left(\int_{0}^{t}\left\|k_{2}(t, \tau)\right\|^{2}\left\|f_{2}(\tau, x(\tau))\right\|_{L^{2}}^{2} d F(\tau)\right)^{\frac{1}{2}} .
$$

THEOREM 3.1. - Let the functions $f_{i}, i=1,2$, in the stochastic functionalintegral equation (1.1), be sublinear, i.e.

(1) $\left|f_{i}(t, x(t ; \omega))\right| \leqslant u_{i}(t)|x(t ; \omega)|+v_{i}(t) \quad P$-a.s. , $\quad i=1,2 ;$

where nonnegative functions $u_{i}$ and $v_{i}, i=1,2$, are continuous and defincd for $t \in R_{+}$, and let us denote

$$
\begin{aligned}
A=\sup \left\{p ( t ) \left\{\int_{0}^{t}\left\|k_{1}(t, \tau)\right\|\left(u_{1}(\tau) \mid p(\tau)\right) d \tau+\right.\right. & \\
& \left.\left.+\left(2 \int_{0}^{t}\left\|k_{2}(t, \tau)\right\|^{2}\left(u_{2}(\tau) / p(\tau)\right)^{2} d F(\tau)\right)^{\frac{1}{2}}\right\}: t \in R_{+}\right\}, \\
B(t)=p(t)\left[|h(t, 0)|+\int_{0}^{t}\|\| k_{1}(t, \tau) \| v_{1}(\tau) d \tau+\right. & \\
& \left.+\left(2 \int_{0}^{t}\left\|k_{2}(t, \tau)\right\|^{2} v_{2}^{2}(\tau) d F(\tau)\right)^{\frac{1}{2}}\right], \quad \text { for } t \in R_{+},
\end{aligned}
$$

$B=\sup \left[B(t): t \in R_{+}\right]$ 
Suppose that

(2) $\lim _{t \rightarrow \infty} B(t)=0$,

(3) $|h(t, x(t ; \omega))-h(t, y(t ; \omega))| \leqslant k|x(t ; \omega)-y(t ; \omega)| P$-a.s. , $\quad k \in[0,1)$;

(4) $M:=A+k<1$;

(5) for any given but fixed $T>0$

$\lim _{\varepsilon \rightarrow 0} \sup \left[\|h(t, x(s))-h(s, x(s))\|_{L^{2}}: s, t \in[0, T],|t-s| \leqslant \varepsilon\right]=0$

uniformly with respect to $x \in U \subset K(\theta, r)$, where $r=B /(1-M)$,

(6) the mappings $x(t ; \omega) \rightarrow f_{i}(t, x(t ; \omega)), i=1,2$, from $C_{p}\left(R_{+}, L^{2}(\Omega, A, P), p\right)$ into $C_{p}\left(R_{+}, L^{2}(\Omega, A, P), p\right)$ are continuous in the topology generated by the norm $\|x\|_{p}$;

(7) $\lim _{i \rightarrow \infty} p(t)\left\|f_{i}(t, x)-f_{i}(t, y)\right\|_{L^{2}}=0, \quad i=1,2$, uniformly with respect to $x$ and $y$ belonging to $K(\theta, r), r=B /(1-M)$;

(8) $m\left(\int_{0}^{t} k_{1}(t, \tau ; \omega) f_{1}(\tau, U(\tau)) d \tau\right) \leqslant L_{1} m(U(t))$,

$$
\begin{aligned}
& m\left(\int_{0}^{t} k_{2}(t, \tau ; \omega) f_{2}(\tau, U(\tau)) d \beta(\tau ; \omega)\right) \leqslant L_{2} m(U(t)), \\
& m(h(t, U(t))) \leqslant L_{3} m(U(t)), \quad L_{i} \in[0,1), i=1,2,3, \\
& U(t)=\left\{x(t) \in L^{2}(\Omega, \mathcal{A}, P): x \in U\right\}, \quad U \subset K(\theta, r), r=B /(1-M) .
\end{aligned}
$$

Then there exists at least one solution $x \in C_{p}$ of (1.1) such that

$$
\lim _{t \rightarrow \infty} p(t)\|x(t)\|_{L^{2}}=0
$$

Proof. - First we note that (3.2), under the assumption (1), takes the form

$$
\begin{aligned}
& \left\|\int_{0}^{t} k_{2}(t, \tau) f_{2}(\tau, x(\tau)) d \beta(\tau)\right\|_{L^{2}} \leqslant \\
& \quad \leqslant \sqrt{2}\left[\|x\|_{\nu}\left(\int_{0}^{t}\left\|k_{2}(t, \tau)\right\|^{2}\left(u_{2}(\tau) / p(\tau)\right)^{2} d F(\tau)\right)^{\frac{1}{2}}+\left(\int_{0}^{t}\left\|k_{2}(t, \tau)\right\|^{2} v_{2}^{2}(\tau) d F(\tau)\right)^{\frac{1}{2}}\right] .
\end{aligned}
$$

Define the map $H$ on $O_{p}$ by

$$
\begin{aligned}
(H x)(t ; \omega)=h(t, x(t ; \omega))+\int_{0}^{t} k_{1}(t, \tau ; \omega) f_{1}(\tau, x(\tau ; \omega)) d \tau+ & \\
& +\int_{0}^{t} k_{2}(t, \tau ; \omega) f_{2}(\tau, x(\tau ; \omega)) d \beta(\tau ; \omega) .
\end{aligned}
$$


Using now (1), (3), (3.1), and (3.3) we get for $x \in O_{p}$

$$
\begin{aligned}
& p(t)\|(H x)(t)\|_{L^{2}} \leqslant p(t)\left\{k\|x(t)\|_{L^{2}}+|h(t, 0)|+\int_{0}^{t}\left\|k_{1}(t, \tau)\right\|\left\|f_{1}(\tau, x(\tau))\right\|_{{ }^{2} \tau} d \tau+\right. \\
& \left.+\left(\int_{0}^{t}\|\| k_{2}(t, \tau)\|\|^{2}\left\|f_{2}(\tau, x(\tau))\right\|_{L^{2}}^{2} d F(\tau)\right)^{\frac{1}{2}}\right\} \leqslant k\|x\|_{p}+p(t)\{|h(t, 0)|+ \\
& +\int_{0}^{t}\left\|k_{1}(t, \tau)\right\| u_{1}(\tau)\|x(\tau)\|_{L^{2}} d \tau+\int_{0}^{t}\|\| k_{1}(t, \tau) \| v_{1}(\tau) d \tau+ \\
& \left.+\sqrt{2}\left(\left(\int_{0}^{t}\left\|k_{2}(t, \tau)\right\|^{2} u_{2}^{2}(\tau)\|x(\tau)\|_{L^{2}}^{2} d F^{\prime}(\tau)\right)^{\frac{1}{2}}+\left(\int_{0}^{t}\left\|k_{2}(t, \tau)\right\|^{2} v_{2}^{2}(\tau) d F(\tau)\right)^{\frac{1}{2}}\right)\right\} \leqslant \\
& \leqslant p(t)\left\{|h(t, 0)|+\int_{0}^{t}\left\|k_{1}(t, \tau)\right\| v_{1}(\tau) d \tau+\sqrt{2}\left(\int^{t}\left\|k_{2}(t, \tau)\right\|^{2} v_{2}^{2}(\tau) d F^{\prime}(\tau)\right)^{\frac{1}{2}}\right\}+ \\
& +\|x\|_{p}\left\{k+p(t) \int_{0}^{0} \int_{0}^{t}\left\|k_{1}(t, \tau)\right\|\left(u_{1}(\tau) / p(\tau)\right) d \tau+\right. \\
& \left.\left.+\sqrt{2}\left(\int_{0}^{t}\|\| k_{2}(t, \tau) \|^{2}\left(u_{2}(\tau) / p(\tau)\right)^{2} d F(\tau)\right)^{\frac{1}{t}}\right]\right\},
\end{aligned}
$$

or we have

$$
\|H x\|_{p} \leqslant M\|x\|_{p}+B
$$

Thus we have proved that $H$ maps $C_{p}$ into $C_{p}$, and moreover, we see that $H$ maps a ball $K(\theta, r)$ into $K(\theta, r)$ with $r=B /(1-M)$.

We now prove that the map $H$ is continuous in the ball $K(\theta, r)$. Let $x, y \in K(\theta, r)$. By (7) for any given $\varepsilon_{i}>0, i=1,2$, we can choose $T>0$ such that

$$
p(\tau)\left\|f_{i}(\tau, x(\tau))-f_{i}(\tau, y(\tau))\right\|_{L^{2}}<\varepsilon_{i}, \quad \text { whenever } \tau>T .
$$

On the other hand, by (2), for any given $\varepsilon^{(i)}>0, i=1,2$, there exist $\delta_{i}>0$, $i=1,2$, such that for all $\tau \in[0, T]$

$$
\begin{aligned}
& p(\tau)\left\|f_{i}(\tau, x(\tau))-f_{i}(\tau, y(\tau))\right\|_{L^{2}}<\varepsilon^{(i)}, \quad i=1,2, \\
& \text { whenever } \quad\|x-y\|_{p}<\delta_{i}, \quad i=1,2 .
\end{aligned}
$$

Moreover, by (3), for any given $\varepsilon_{3}>0$ there exists $\delta>0$ such that

$$
p(t)\|h(t, x(t))-h(t, y(t))\|_{L^{2}}<\varepsilon_{3}, \quad \text { whenever } \quad\|x-y\|_{p}<\delta .
$$

Furthermore, we can assume without loss of generality that there exists $T>0$ such that $u_{i}(t) \geqslant 1, i=1,2$, whenever $t \geqslant T$, and $\min \left\{u_{i}(\tau): 0 \leqslant \tau \leqslant T\right\}=u_{T}^{i}>0, i=1,2$. 
Hence, using (3.8), and putting $\max \{p(\tau): 0 \leqslant \tau \leqslant T\}=p_{T}$, we have for $t \geqslant T$

$$
\begin{aligned}
& p(t)\|(H x)(t)-(B y)(t)\|_{\pi^{2}} \leqslant p(t)\left\{\|h(t, x(t))-h(t, y(t))\|_{L^{2}}+\right. \\
& +\int_{0}^{t}\left\|k_{1}(t, \tau)\right\|\|\|_{1}(\tau, x(\tau))-f_{1}(\tau, y(\tau)) \|_{L^{2}} d \tau+\left(\int_{0}^{t}\left\|k_{2}(t, \tau)\right\|\left\|^{2}\right\| f_{2}(\tau, x(\tau))-\right. \\
& \left.\left.-f_{2}(\tau, y(\tau)) \|_{L^{2}}^{2} d F(\tau)\right)_{t}^{\frac{1}{2}}\right\} \leqslant \varepsilon_{3}+p(t)\left\{\left(p_{T} / u_{1}^{p}\right) \int_{0}^{T}\left(\left\|k_{c_{1}}(t, \tau)\right\| u_{1}(\tau) / p(\tau)\right) p(\tau) \cdot \| f_{1}(\tau, x(\tau))-\right. \\
& -f_{1}(\tau, y(\tau))\left\|_{L^{2}} d \tau+\int_{T}^{t}\left(\left\|k_{1}(t, \tau)\right\| u_{1}(\tau) / p(\tau)\right) p(\tau)\right\| f_{1}(\tau, x(\tau))-f_{1}(\tau, y(\tau)) \|_{L^{2}} d \tau+ \\
& +\left(p_{T} / u_{2}^{T}\right)\left(\int_{0}^{T}\left\|k_{2}(t, \tau)\right\|_{t}{ }^{2}\left(u_{2}(\tau) / p(\tau)\right)^{2} p^{2}(\tau) \cdot\left\|f_{2}(\tau, x(\tau))-f_{2}(\tau, y(\tau))\right\|_{L^{2}}^{2} d F(\tau)\right)^{\frac{1}{2}}+ \\
& \left.+\left(\int_{\tau}^{t}\left\|\tilde{k}_{2}(t, \tau)\right\|^{2}\left(u_{2}(\tau) / p(\tau)\right)^{2} p^{2}(\tau) \cdot\left\|f_{2}(\tau, x(\tau))-f_{2}(\tau, y(\tau))\right\|_{L^{2}}^{2} d F(\tau)\right)^{\frac{1}{2}}\right\} \text {. }
\end{aligned}
$$

Therefore, by (4), (3.6), (3.7), and (3.9), we obtain

$$
\sup _{t \geqslant T} p(t) \|(H x)(t)-\left.(H y)(t)\right|_{L^{2}} \leqslant \varepsilon_{3}+M\left(\left(p_{T} / u_{1}^{T^{T}}\right) \varepsilon^{(1)}+\varepsilon_{1}+\left(p_{T} / u_{2}^{T}\right) \varepsilon^{(2)}+\varepsilon_{2}\right)
$$

Moreover, it can be seen that for any given $\varepsilon_{4}>0$ one has

$$
\underset{0 \leqslant t \leqslant T}{\sup p(t)}\|(H x)(t)-(H y)(t)\|_{L^{2}}<\varepsilon_{4}, \quad \text { whenever } \quad\|x-y\|_{p}<\delta
$$

Thus, by (3.10) and (3.11), for any given $\varepsilon>0$

$$
\|H x-H y\|_{p}<\varepsilon, \quad \text { whenever } \quad\|x-y\|_{p}<\delta, \quad x, y \in K(\theta, r) .
$$

Let now be given $\varepsilon>0, T>0$ and $t, s \in[0, T],|t-s|<\varepsilon$. By (3.4) for $0 \leqslant s<t$ and $x \in U \subset K(\theta, r)$, we have

$$
\begin{aligned}
& -h(s, x(s))\left\|_{L^{2}}+|p(t)-p(s)| \cdot\right\| \int_{0}^{t} k_{1}(t, \tau) f_{1}(\tau, x(\tau)) d \tau \|_{L^{2}}+ \\
& +p(s)\left\|\int_{0}^{s}\left(k_{1}(t, \tau)-k_{1}(s, \tau)\right) f_{1}(\tau, x(\tau)) d \tau_{L^{2}}+p(s)\right\| \int_{0}^{t} k_{1}(t, \tau) f_{1}(\tau, x(\tau)) d \tau \|_{L^{2}}+ \\
& +|p(t)-p(s)|\left\|\int_{0}^{t} k_{2}(t, \tau) f_{2}(\tau, x(\tau)) d \beta(\tau)\right\|_{L^{2}}^{+}+ \\
& +p(s)\left\|\int_{0}^{s}\left(k_{2}(t, \tau)-k_{2}(s, \tau)\right) f_{2}(\tau, x(\tau)) d \beta(\tau)\right\| L_{L^{2}}+p(s)\left\|\int_{s}^{t} k_{2}(t, \tau) f_{2}(\tau, x(\tau)) d \beta(\tau)\right\|_{L^{2}}
\end{aligned}
$$


But using (3.1) with $i=1$ and $x$ replaced by $f_{1}(\tau, x(\tau))$, we obtain

$$
\begin{aligned}
& |p(t)-p(s)|\left\|\int_{0}^{t} k_{1}(t, \tau) f_{1}(\tau, x(\tau)) d \tau\right\|_{L^{2}} \leqslant \\
& \qquad|p(t)-p(s)| \int_{0}^{t}\left\||| k_{1}(t, \tau)\right\|\|\| u_{1}(\tau)|x(\tau)|+v_{1}(\tau) \|_{L^{2}} d \tau \leqslant \\
& \leqslant T|p(t)-p(s)|\left(r \max \left\{\|\| k_{1}(t, \tau) \|\left(u_{1}(\tau) \mid p(\tau)\right): 0 \leqslant \tau \leqslant T\right\}+\right. \\
& \left.\quad+\max \left\{\mid\left\|k_{1}(t, \tau)\right\| v_{1}(\tau): 0 \leqslant \tau \leqslant T\right\}\right) .
\end{aligned}
$$

Similarly, we get

$$
\begin{aligned}
& p(s) \| \int_{0}^{s}\left(k_{1}(t, \tau)-\right.\left.k_{1}(s, \tau)\right) f_{1}(\tau, x(\tau)) d \tau \|_{L^{2}} \leqslant \\
& \leqslant \operatorname{Tr} p(s) \max \left\{\left\|k_{1}(t, \tau)-k_{1}(s, \tau)\right\|\left(u_{1}(\tau) / p(\tau)\right): 0 \leqslant \tau \leqslant T\right\}+ \\
&+T p(s) \max \left\{\left\|k_{1}(t, \tau)-k_{1}(s, \tau) \cdot\right\| v_{1}(\tau): 0 \leqslant \tau \leqslant T\right\}
\end{aligned}
$$

and

$$
\begin{aligned}
& p(s)\left\|\int_{s}^{t} k_{1}(t, \tau) f_{1}(\tau, x(\tau)) d \tau\right\|_{L^{2}} \leqslant \\
& \leqslant|t-s| p(s)\left(r \max \left\{\left\|k_{1}(t, \tau)\right\|\left(u_{1}(\tau) / p(\tau)\right): 0 \leqslant \tau \leqslant T\right\}+\right. \\
& \\
&\left.+\max \left\{\left\|k_{1}(t, \tau)\right\| v_{\mathbf{1}}(\tau): 0 \leqslant \tau \leqslant T\right\}\right) .
\end{aligned}
$$

Now using (3.2) and (3.3), we have the following estimates

$$
\begin{aligned}
|p(t)-p(s)|\left\|\int_{0}^{t} k_{2}(t, \tau) f_{2}(\tau, x(\tau)) d \beta(\tau)\right\| L_{L^{2}} \leqslant \sqrt{2}(F(T)-F(0))^{\frac{\pi}{2}}|p(t)-p(s)| \cdot \\
\cdot\left[r\left(\max \left\{\left\|k_{2}(t, \tau)\right\| \|^{2}\left(J_{2}(\tau) / p(\tau)\right)^{2}: 0 \leqslant \tau \leqslant T\right\}\right)^{\frac{1}{2}}+\right.
\end{aligned}
$$$$
\left.+\left(\max \left\{\|\| k_{2}(t, \tau) \mid \|^{2} v_{2}^{2}(\tau): 0 \leqslant \tau \leqslant T\right\}\right)^{\frac{1}{2}}\right],
$$

$$
\begin{aligned}
p(s)\left\|\int_{0}^{s}\left(k_{2}(t, \tau)-k_{2}(s, \tau)\right) f_{2}(\tau, x(\tau)) d \beta(\tau)\right\|_{L^{2}} \leqslant \sqrt{2} p(s)(F(T)-F(0))^{\frac{1}{2}} \\
\cdot\left[r\left(\max \left\{\left\|k_{2}(t, \tau)-k_{2}(s, \tau)\right\| \|^{2}\left(u_{2}(\tau) / p(\tau)\right)^{2}: 0 \leqslant \tau \leqslant T\right\}\right)^{\frac{1}{2}}+\right. \\
\left.+\left(\max \left\{\left\|k_{2}(t, \tau)-k_{2}(s, \tau)\right\|^{2} v_{2}^{2}(\tau): 0 \leqslant \tau \leqslant T\right\}\right)^{\frac{1}{2}}\right]
\end{aligned}
$$

and

$$
\begin{aligned}
& p(s)\left\|\int_{s} k_{2} k_{2}(t, \tau) f_{2}(\tau, x(\tau)) d \beta(\tau)\right\|_{L^{2}} \leqslant \sqrt{2} p(s)(F(t)-F(s))^{\frac{1}{3}} . \\
& \cdot\left[r\left(\max \left\{\|\| k_{2}(t, \tau) \|^{2}\left(u_{2}(\tau) / p(\tau)\right)^{2}: 0 \leqslant \tau \leqslant T\right\}\right)^{\frac{1}{t}}+\right. \\
&\left.+\left(\max \left\{\left\|k_{2}(t, \tau)\right\|^{2} v_{2}^{2}(\tau): 0 \leqslant \tau \leqslant T\right\}\right)^{3}\right]
\end{aligned}
$$


We need to recall the definition of the modulus of continuity which is defined for a real function $w$ as:

$$
\nu_{T}(w ; \varepsilon)=\sup \{|w(t)-w(s)|: t, s \in[0, T],|t-s| \leqslant \varepsilon\}, \quad \varepsilon>0 .
$$

Now, the properties of the functions $p, k_{1}, k_{2}$, and $F$ allow us to write

$$
\lim _{\varepsilon \rightarrow 0} \nu_{T}(p ; \varepsilon)=\lim _{\varepsilon \rightarrow 0} \nu_{T}\left(\left\|k_{1}\right\| ; \varepsilon\right)=\lim _{\varepsilon \rightarrow 0} \nu_{x}\left(\|\| k_{2} \| ; \varepsilon\right)=\lim _{\varepsilon \rightarrow 0} \nu_{T}(F ; \varepsilon)=0
$$

Moreover, by the assumptions (3) and (5), we have

$$
\lim _{\delta \rightarrow 0} v_{T}\left(\|h(t, x(t))-h(s, x(s))\|_{L^{2}} ; \varepsilon\right)=0
$$

Therefore, by (3.12)-(3.18), and (3.20), (3.21), we get

$$
\beta_{0}((H U))=0 \text {. }
$$

Fix now $U \subset K(\theta, r), r=B /(1-M)$. We prove that

$$
a((H U)) \leqslant M a(U) .
$$

It is clear, by the definition of the integral, that for any given $\eta_{1}>0$ there exists a positive integer $n_{1}=n_{1}\left(\eta_{1}\right)$ such that for $n \geqslant n_{1}$

$$
\begin{aligned}
\mid \int_{0}^{t}\left\|k_{1}(t, \tau)\right\|\left(u_{1}(\tau)\|x(\tau)\|_{L^{2}} / p(\tau)\right) p(\tau) d \tau- & \\
& -\sum_{k=0}^{n-1} \frac{t}{n}\left\|\left|k_{1}\left(t, \frac{k t}{n}\right) \|\right|\left(u_{1}\left(\frac{k t}{n}\right)\left\|x\left(\frac{k t}{n}\right)\right\|_{L^{2}} / p\left(\frac{k t}{n}\right)\right) p\left(\frac{k t}{n}\right) \mid<\eta_{1} .\right.
\end{aligned}
$$

Let now $T<i$. Put $k_{1}^{*}=\min [k: 0 \leqslant k \leqslant n, k t / n<T]$.

Then we have

$$
\begin{aligned}
& \int_{0}^{t}\left\|k_{1}(t, \tau)\right\|\left(u_{1}(\tau)\|x(\tau)\|_{L^{2}} / p(\tau)\right) p(\tau) d \tau \leqslant \\
& \quad \leqslant \eta_{1}+\sum_{k=0}^{k_{1}^{*}} \frac{t}{n}\|\| k_{1}\left(t, \frac{k t}{n}\right) \|\left(u_{1}\left(\frac{k t}{n}\right)\left\|x\left(\frac{k t}{n}\right)\right\|_{L^{2}} / p\left(\frac{k t}{n}\right)\right) p\left(\frac{k t}{n}\right)+ \\
& \quad+\sum_{k=k_{1}^{*}+1}^{n-1} \frac{t}{n}\|\|_{1_{1}}\left(t, \frac{k t}{n}\right) \|\left(u_{1}\left(\frac{k t}{n}\right)\left\|x\left(\frac{k t}{n}\right)\right\|_{L^{2}} / p\left(\frac{k t}{n}\right)\right) p\left(\frac{k t}{n}\right)=\eta_{1}+I_{1}+I_{2} .
\end{aligned}
$$

Now for any given $\eta_{2}>0$ there exists $n_{2}=n\left(\eta_{2}\right)$ such that for $n \geqslant n_{2}$

$$
I_{1} \leqslant k_{1}^{*} t \max \left[p\left(\frac{k t}{n}\right)\left\|x\left(\frac{k t}{n}\right)\right\|_{L^{2}}: \frac{k t}{n}<T\right] \max \left\{\left\|k_{1}(t, \tau)\right\|\left(u_{1}(\tau) / p(\tau)\right): 0 \leqslant \tau \leqslant T\right\} / n<\eta_{2} .
$$


Similarly, for any given $\eta_{3}>0$

$$
I_{2} \leqslant \sup \left\{p(t)\|x(t)\|_{L^{2}}: t \geqslant T\right\}\left(\int_{0}^{t}\left\|k_{1}(t, \tau)\right\|\left(u_{1}(\tau) / p(\tau)\right) d \tau+\eta_{3}\right)
$$

for sufficiently large $n$.

Further, by the definition of the Stieltjes integral, for any given $\eta_{1}^{\prime}$

$$
\begin{aligned}
& \mid \int_{0}^{t}\|\| k_{2}(t, \tau) \|^{2}\left(u_{2}(\tau)\|x(\tau)\|_{L^{2}} / p(\tau)\right)^{2} p^{2}(\tau) d F(\tau)- \\
& \left.-\left.\sum_{k=0}^{n-1}\left(F\left(\frac{(k+1) t}{n}\right)-F\left(\frac{k t}{n}\right)\right)||\left|k_{2}\left(t, \frac{k t}{n}\right)\right||| u^{2}\left(\frac{n}{k t}\right)|| \infty\left(\frac{k t}{n}\right)\right|_{L^{2}} \mid p\left(\frac{k t}{n}\right)\right)^{2} p^{2}\left(\frac{k t}{n}\right) \mid<\eta_{1}^{\prime}
\end{aligned}
$$

for sufficiently large $n$.

Let now $T<t$. Put $k_{2}^{*}=\min [k: 0 \leqslant k \leqslant n, k t / n<T]$.

Then for any given $\eta_{1}^{\prime}$ we have

$$
\begin{gathered}
\int_{0}^{t} \| k_{2}\left(t, \tau \|^{2}\left(u_{2}(\tau)\|x(\tau)\|_{L^{2}}(p(\tau))^{2} p^{2}(\tau) d F^{\prime}(\tau) \leqslant \eta_{1}+\right.\right. \\
+\sum_{k=0}^{k_{2}^{*}}\left(F\left(\frac{(k+1) t}{n}\right)-F^{\prime}\left(\frac{k t}{n}\right)\right)\left\|k_{2}\left(t, \frac{k t}{n}\right)\right\|\left(u_{2}\left(\frac{k t}{n}\right)\left\|x\left(\frac{k t}{n}\right)\right\|_{L^{2}} / p\left(\frac{k t}{n}\right)\right)^{2} p^{2}\left(\frac{k t}{n}\right)+ \\
+\sum_{k=k^{*}+1}^{n-1}\left(F\left(\frac{(k+1) t}{n}\right)-F\left(\frac{k t}{n}\right)\right)\left\|\mid k_{2}\left(t, \frac{k t}{n}\right)\right\|\left(u_{2}\left(\frac{k t}{n}\right)\left\|x\left(\frac{k t}{n}\right)\right\|_{L^{2}} / p\left(\frac{k t}{n}\right)\right)^{2} \cdot \\
\cdot p^{2}\left(\frac{k t}{n}\right)=\eta_{1}^{\prime}+I_{1}^{\prime}+I_{2}^{\prime} .
\end{gathered}
$$

Now by the continuity of $F$ for any given $\eta_{2}^{\prime}>0$ we have

$$
\begin{aligned}
I_{1}^{\prime} \leqslant \max _{0 \leqslant k \leqslant k_{2}^{*}}\left(F^{\prime}\left(\frac{(k+1) t}{n}\right)-\eta\left(\frac{k t}{n}\right)\right) \max & \left\{p^{2}\left(\frac{k t}{n}\right)\left\|x\left(\frac{k t}{n}\right)\right\|_{L^{2}}^{2}: \frac{k t}{n} \leqslant T\right\} \\
& \cdot \max \left\{\left\|k_{2}(t, \tau)\right\|^{2}(u(\tau) / p(\tau))^{2}: 0 \leqslant \tau \leqslant T\right\}<\eta_{2}^{\prime}
\end{aligned}
$$

for sufficiently large $n$.

Similarly, for any given $\eta_{3}^{\prime}>0$

$$
I_{2}^{\prime} \leqslant\left(\sup \left\{p(t)\|x(t)\|_{L^{2}}: t \geqslant T^{\prime}\right)^{2}\left(\int_{0}^{t}\|\| k_{2}(t, \tau) \|^{2}\left(u_{2}(\tau) / p(\tau)\right) d F(\tau)+\eta_{3}^{\prime}\right)\right.
$$

for sufficiently large $n$.

Moreover, we note that by (3)

$$
p(t)\|h(t, x(t))\|_{L^{2}} \leqslant k \sup \left[p(t)\|x(t)\|_{L^{2}}: t>T\right]+p(t)|h(t, 0)| .
$$


Therefore, by the above considerations, we get

$$
\begin{aligned}
& p(t)\|(H x)(t)\|_{L^{2}} \leqslant p(t)[h(t ; 0)\}+M \sup \left\{p(t)\|x(t)\|_{L^{2}}: t \geqslant T\right\}+ \\
&+p(t)\left[\eta_{1}+\eta_{2}+\sqrt{\eta_{1}^{\prime}}+\sqrt{\eta_{2}^{\prime}}+\left(\eta_{3}+\sqrt{\eta_{3}^{\prime}}\right) r\right]+p(t) \int_{0}^{t}\left\|k_{1}(t, \tau)\right\| v_{1}(\tau) d \tau+ \\
&+\sqrt{2} p(t)\left(\int_{0}^{t}\left\|k_{2}(t, \tau)\right\| v_{2}^{2}(\tau) d F(\tau)\right)^{\frac{1}{2}}
\end{aligned}
$$

Thus, by the assumptions of Theorem 3.1, we obtain

$$
\begin{aligned}
\lim _{T \leftarrow \infty} \sup _{x \in U}\left[\operatorname { s u p } \left\{p(t)\|(H x)(t)\|_{L^{2}}: \dot{t}\right.\right. & \geqslant T\}] \leqslant\left(\eta_{1}+\eta_{2}+\sqrt{\eta_{1}^{\prime}}+\sqrt{\eta_{2}^{\prime}}+\right. \\
& \left.+\left(\eta_{3}+\sqrt{\eta_{3}^{\prime}}\right) \eta\right) O+M \lim _{T \rightarrow \infty}\left[\sup _{x \in U}\left(\sup \left\{p(t)\|x(t)\|_{L^{2}}: t \geqslant T\right\}\right)\right] .
\end{aligned}
$$

Let $\eta_{i} \rightarrow 0, \eta_{i}^{\prime} \rightarrow 0, i=1,2,3$. Then we get (3.23). Finally, by (3.22), (3.23), and the assumptions (8) we obtain

$$
\mu_{0}(H U) \leqslant D \mu_{0}(U)
$$

where $D=\max \left\{L_{1}, L_{2}, L_{3}, M\right\}$, which proves that $H$ is a $\mu_{0}$-contraction. This fact by Theorem 2.1 ends the proof.

\section{4. - An application.}

Consider the stochastic control system described by

$$
d x(t ; \omega)=A(\omega) x(t ; \omega) d t+f_{1}(t, \delta(t ; \omega)) d t+f_{2}(t, \delta(t ; \omega)) d \beta(\tau)
$$

with

$$
\delta(t ; \omega)=h(t, \delta(t ; \omega))+\langle G, x(t ; \omega)\rangle
$$

where

(a) $x(t ; w)$ is the unknown $n \times 1$ vector random process;

(b) $\delta(t ; \omega)$ is the unknown random process (error signal);

(c) $A(\omega)$ is $n \times n$ random matrix;

(d) $f_{1}(t, \delta)$ and $f_{2}(t, \delta)$ are $n \times 1$ vector functions and $h(t, \delta)$ is a scalar function;

(e) $\theta$ is a constant $1 \times n$ vector;

(f) $\beta(\tau)$ is a standard Brownian motion process. 
In what follows $\langle\cdot, \cdot\rangle$ will denote the inner product and $|\cdot|$, a vector or a matrix norm. One can show that the above random system can be expressed in the following integral form

$$
\begin{aligned}
\delta(t ; \omega)=h(t, \delta(t ; \omega))+\left\langle G, \exp [A(\omega) t] x_{0}(\omega)\right\rangle+ & \\
& +\int_{0}^{t}\left\langle G, \exp [A(\omega)(t-\tau)] f_{1}(\tau, \delta(\tau ; \omega))\right\rangle d \tau+ \\
& +\int_{0}^{t}\left\langle G, \exp [A(\omega)(t-\tau)] f_{2}(\tau, \delta(\tau ; \omega))\right\rangle d \beta(\tau) .
\end{aligned}
$$

In [7] there are given sufficient conditions for the stochastic system (4.1)-(4.2) under which

$$
E\left[|\delta(t ; \omega)|^{2}\right] \rightarrow 0, \quad t \rightarrow \infty
$$

We now give a theorem informing us on solutions of (4.1)-(4.2) under weaker assumptions than those of [7]. Some corollaries of this theorem allow to prove (4.4) under conditions which are more general than those of [7], and can be easily verified.

THEOREM 4.1. - Let the functions $f_{i}, i=1,2$, in the stochastic functionalintegral equation (4.3), be sublinear, i.e.

$$
\left|f_{i}(t, \delta(t ; \omega))\right| \leqslant u_{i}(t)|\delta(t ; \omega)|+v_{i}(t) P \text {-a.s. }
$$

where nonnegative functions $u_{i}$ and $v_{i}, i=1,2$, are continuous and defined for $t \in R_{+}$, and let us denote

$$
\begin{aligned}
& A=\sup \left\{p ( t ) \left\{|G| \int_{0}^{t}\|\exp (A(t-\tau))\|\left(u_{1}(\tau) / p(\tau)\right) d \tau+\right.\right. \\
& \left.\left.+\left(2 \int_{0}^{t}\|\exp (A(t-\tau))\|^{2}\left(u_{2}(\tau) / p(\tau)\right)^{2} d \tau\right)^{\frac{1}{2}}\right\}: t \in R_{+}\right\}, \\
& B(t)=p(t)\left[|h(t, 0)|+\int_{0}^{t}\|\exp (A(t-\tau))\| v_{1}(\tau) d \tau+\right. \\
& \left.+\left(2 \int_{0}^{t}\|\exp (A(t-\tau))\| \|^{2} v_{2}^{2}(\tau) d \tau\right)^{\frac{1}{2}}\right], \quad t \in R_{+},
\end{aligned}
$$

$B=\sup \left[B(t): t \in R_{+}\right]$

Suppose that

$$
\lim _{b \rightarrow \infty} B(t)=0
$$


(3) $\left|h\left(t, \delta_{1}(t ; \omega)\right)-h\left(t, \delta_{2}(t ; \omega)\right)\right| \leqslant k\left|\delta_{1}(t ; \omega)-\delta_{2}(t ; \omega)\right| P-$ a.s. , $\quad k \in[0,1)$,

$$
M:=A+k<1,
$$

for any given but fixed $T>0$

(5) $\lim _{\varepsilon \rightarrow 0} \sup \left[\|h(t, \delta(s))-h(s, \delta(s))\|_{L^{2}}: t, s \in[0, T], \mid t-s t \leqslant \varepsilon\right]=0$

uniformly with respect to $\delta \in U \subset K(\theta, r)$, where $r=B /(1-M)$,

(6) the mappings $\delta(t ; \omega) \rightarrow f_{i}(t, \delta(t ; \omega)), i=1,2$, from $C_{p}\left(R_{+}, L^{2}(\Omega, \mathcal{A}, P), p\right)$ into $C_{p}\left(R_{+}, L^{2}(\Omega, \mathcal{A}, P), p\right)$ are continuous in the topology generated by norm $\|\delta\|_{0}$,

$$
\lim _{t \rightarrow \infty} p(t)\left\|f_{i}\left(t, \delta_{1}\right)-f_{i}\left(t, \delta_{2}\right)\right\|_{L^{2}}=0, \quad i=1,2,
$$

uniformly with respect to $\delta_{1}$ and $\delta_{2}$ belonging to $K(\theta, r), r=B /(1-M)$,

$$
\text { (8) } \begin{aligned}
& m\left(\int_{0}^{t}\left\langle G, \exp [A(\omega)(t-\tau)] f_{1}(\tau, U(\tau))\right\rangle d \tau\right) \leqslant L_{1} m(U(t)), \\
& m\left(\int_{0}^{t}\left\langle G, \exp [A(\omega)(t-\tau)] f_{2}(\tau, U(\tau))\right\rangle d \beta(\tau)\right) \leqslant L_{2} m(U(t)), \\
& m(h(t, U(t))) \leqslant L_{3} m(U(t)), \quad L_{i} \in[0,1), \quad i=1,2,3, \\
& U(t)=\left\{\delta(t) \in L^{2}(\Omega, \mathcal{A}, P): \delta \in U\right\}, \quad U \subset K(\theta, r), \quad r=B /(1-M) .
\end{aligned}
$$

Then there exists at least one solution $x \in C_{p}$ of (4.1)-(4.2) such that

$$
\lim _{t \rightarrow \infty} p(t)\|\delta(t)\|_{L^{2}}=0
$$

Proof. - The statement (4.5) can be easily lobtained by the arguments contained in the proof of Theorem 3.1.

REMARK 4.1. - Note that if the functions $f_{i}(t, \delta), i=1,2$, and $h(t, \delta)$ are jointly continuous in $t$ and $\delta$ such that

$$
\left|f_{i}\left(t, \delta_{1}\right)-f_{i}\left(t, \delta_{2}\right)\right| \leqslant k_{i}\left|\delta_{1}-\delta_{2}\right|, \quad i=1,2
$$

and

$$
\left|h\left(t, \delta_{1}\right)-h\left(t, \delta_{2}\right)\right| \leqslant k\left|\delta_{1}-\delta_{2}\right|
$$

and let us denote

$$
p(\tau)=A \exp [-\beta \tau], \quad \text { and } \quad\|\exp (A(t-\tau))\| \leqslant L \exp (-\alpha(t-\tau)),
$$

where $k, k_{i}, \Lambda, L$ are positive constants, and $0<\beta<\alpha$, then the assumptions of Theorem 4.1 are satisfied. 


\section{REFERENCES}

[1] J. Banas, Measure of Noncompactness in the Space of Continuous Tempered Functions, Demonstratio Math., 14 (1981), pp. 127-133.

[2] J. Banaś - K. GoEBeL, Measure of Noncompactness in Banach Space, Lecture Notes in Pure and Applied Mathematics, Marcel Dekker, Inc. Vol. 60 (1980), New York and Basel.

[3] A. T. Bharucha-Reid, Random Integral Equations, Academic Press, New York, N. Y., 1972 .

[4] A. T. Bhardcha-ReID, Lectures on Theory of Random Equations, Madras, 1963.

[5] G. Darbo, Punti Uniti in Transformazioni a Codominio non Compatto, Rend. Sem. Mat. Univ. Padova, 24 (1955), pp. 84-92.

[6] J. L. Dooв, Stochastic Processes, John Wiley and Sons, Inc., New York, N. Y., 1953.

[7] M. N. Manodgian - A. N. V. RAO - C. P. Tsokos, On a Nonlinear Stochastic Integral Equation with Application to Control Systems, Ann. di Math. Pura ed App. (IV), Vol. CX, (1976), pp. 211-222.

[8] W. J. Padgert, On a Stochastic Integral Equation of the Volterra Type in Telephone Traffic Theory, Journal of Applied Probability, 8 (1971), pp. 269-275.

[9] A. N. V. RAO - C. P. Tsokos, On a Class of. Stochastic Functional-Integral Equations, Coll. Math., 35, 1 (1976), pp. 141-146.

[10] A. V. SkokoкноD, Studies in the Theory of Random Processes, Addison-Wesley, Reading, Massachusetts, 1965.

[11] A. V. SкококноD, Stokhastičeskie uravnienia dla sloznykh sistem, Moskva, 1983 (in Rus$\operatorname{sian})$.

[12] C. P. Tsokos - W. J. PaDgETt, Random Integral Equations with Applications to Life Sciences and Engineering, Academic Press, New York, N. Y., 1974.

[13] C. P. Tsokos - W. J. PADGETT, Random Integral Equations with Applications to Stochastic Systems, Lecture Notes in Mathematics, Col. 233, Springer-Verlag, Berlin-Heidelberg. New York, 1971.

[14] C. P. Tsokos, On some Nonlinear Differential Systems with Random Parameters, IEEE Proceedings of the Third Annual Princeton Conference on Informatical Sciences and Systems, (1969), pp. 228-234.

[15] K. ZrMA, Sur l'Existence des Solutions d'une Équation Integro-différentielle, Ann. Polon. Math., 27 (1973), pp. $181-187$. 